University of Nebraska - Lincoln

DigitalCommons@University of Nebraska - Lincoln

2005

\title{
Molecular Cloning and Characterization of a Porcine UL16 Binding Protein (ULBP)-Like cDNA
}

\author{
Carmen N. Garcia-Borges \\ University of Arkansas for Medical Sciences, 151 Research, 4300 West 7th Street, Little Rock, Arkansas \\ Bounleut Phanavanh \\ University of Arkansas for Medical Sciences, 151 Research, 4300 West 7th Street, Little Rock, Arkansas \\ Sarika Saraswati \\ University of Arkansas for Medical Sciences, 151 Research, 4300 West 7th Street, Little Rock, Arkansas \\ Richard A. Dennis \\ University of Arkansas for Medical Sciences, 151 Research, 4300 West 7th Street, Little Rock, Arkansas \\ Mark D. Crew \\ University of Arkansas for Medical Sciences, 151 Research, 4300 West 7th Street, Little Rock, Arkansas
}

Follow this and additional works at: https://digitalcommons.unl.edu/publichealthresources

Part of the Public Health Commons

Garcia-Borges, Carmen N.; Phanavanh, Bounleut; Saraswati, Sarika; Dennis, Richard A.; and Crew, Mark D., "Molecular Cloning and Characterization of a Porcine UL16 Binding Protein (ULBP)-Like cDNA" (2005).

Public Health Resources. 19.

https://digitalcommons.unl.edu/publichealthresources/19

This Article is brought to you for free and open access by the Public Health Resources at DigitalCommons@University of Nebraska - Lincoln. It has been accepted for inclusion in Public Health Resources by an authorized administrator of DigitalCommons@University of Nebraska - Lincoln. 


\title{
Molecular cloning and characterization of a porcine UL16 binding protein (ULBP)-like cDNA 年
}

\author{
Carmen N. García-Borges ${ }^{\mathrm{a}, \mathrm{b}}$, Bounleut Phanavanh ${ }^{\mathrm{a}, \mathrm{c}}$, Sarika Saraswati ${ }^{\mathrm{b}}$, \\ Richard A. Dennis ${ }^{\mathrm{d}}$, Mark D. Crew ${ }^{\mathrm{a}, \mathrm{b}, \mathrm{c}, *}$ \\ ${ }^{a}$ Central Arkansas Veterans Healthcare System, University of Arkansas for Medical Sciences, 151 Research, \\ 4300 West 7th Street, Little Rock, Arkansas 72205, USA \\ ${ }^{\mathrm{b}}$ Department of Biochemistry and Molecular Biology, University of Arkansas for Medical Sciences, 151 Research, \\ 4300 West 7th Street, Little Rock, Arkansas 72205, USA \\ ${ }^{c}$ Department of Microbiology and Immunology, University of Arkansas for Medical Sciences, 151 Research, \\ 4300 West 7th Street, Little Rock, Arkansas 72205, USA \\ ${ }^{\mathrm{d}}$ Department of Geriatrics, University of Arkansas for Medical Sciences, 151 Research, 4300 West 7th Street, Little Rock, Arkansas 72205, USA
}

Received 13 August 2004

Available online 10 November 2004

\begin{abstract}
UL16 binding proteins (ULBPs) are ligands for the NK cell activating receptor NKG2D. A cDNA encoding a porcine ULBP-like protein (PULBP) was cloned and the predicted amino acid sequence exhibited 35-52\% identity to human ULBPs. Southern blot analysis suggested that there is only one ULBP-like gene in the pig genome. Transcripts of PULBP and another potential NKG2D ligand, MIC2, were detected by RT-PCR in a wide range of tissues. Recombinant PULBP-Fc and human ULBP2-Fc fusion proteins were made and used to examine PULBP binding to porcine PBMCs and a human NK cell line (NKL cells). PULBP-Fc bound to a subpopulation of porcine PBMCs but not NKL cells. Conversely, human ULBP2-Fc did not bind to porcine PBMCs but did stably interact with NKL cells.
\end{abstract}

Keywords: NKG2D ligand; ULBP; Pig; Natural killer cells

\section{Introduction}

The human UL16 binding proteins (ULBPs) function as activating ligands for the NK cell receptor NKG2D (Cosman et al., 2001; Sutherland et al., 2002). Upon infection with human cytomegalovirus, the viral protein UL16 binds some ULBPs (ULBP1 and ULBP2) thereby preventing their cellsurface expression as one mechanism for immune evasion (Rolle et al., 2003). In humans, four members of this MHC class I-extended gene family have been described (Cosman

\footnotetext{
The nucleotide sequence data reported in this paper have been submitted to the GenBank nucleotide sequence database and have been assigned the accession number AY248037.

* Corresponding author. Tel.: +1 501257 5557; fax: +1 5012574822 .

E-mail address: crewmarkd@uams.edu (M.D. Crew).
}

et al., 2001; Jan et al., 2003). Human ULBP1-3 are GPIlinked molecules while ULBP4 is a transmembrane protein. Members of the MHC class I-extended gene family, human ULBP1-4 contain $\alpha 1$ and $\alpha 2$ domains, but not $\alpha 3$ (and they do not bind $\beta 2$-microglobulin). The expression of these molecules varies; ULBP4 has been reported to be expressed predominantly in the skin (Jan et al., 2003) while other ULBPs are expressed in a wide variety of tissues including lung, liver, heart and testis (Cosman et al., 2001). All four ULBPs are potent ligands for the activating NKG2D receptor found on NK cells, NKT cells, and on a subset of CD8+ T cells. Ligands for NKG2D, like the ULBPs, appear to have significant weight in the balance between activation and inhibition of NK cell activity (reviewed in Vivier et al., 2002). The interaction of ULBPs with NKG2D is capable of activating NK cells 
even in the presence of inhibitory signals (Cosman et al., 2001).

ULBP-like genes have also been identify in the mouse. In mouse, these are known as the Raetl gene family (retinoic acid early transcripts 1 ) and include four highly related genes. Raet1 gene product, RAE-1, and H60, another mouse homologue of ULBP (Cerwenka et al., 2000, 2002) are similar in structure as the ULBPs, containing extracellular domains with distant similarity to the $\alpha 1$ and $\alpha 2$ domains of the MHC class I molecules, but contain a low level of sequence conservation. Both of these proteins have been shown to interact with the mouse NKG2D receptor (Cerwenka et al., 2000; Diefenbach et al., 2001). More recently, two ULBPlike transcripts have been identified in cattle and they have been termed MHCLA1 and MHCLA2.

Human NKG2D also binds another class of MHC class I-extended gene family members, namely the MHC-encoded MICA and MICB proteins. These are also members of the MHC class I gene superfamily but unlike ULBPs, MICA/B contain $\alpha 3$ domains and are anchored to the membrane by transmembrane domains (Vivier et al., 2002). MICA and MICB are stress-inducible proteins whose expression is upregulated in certain tumors and infected cells (Groh et al., 1996, 1999, 2001). Mice apparently lack orthologs of MICA and MICB but the presence of MICA- and MICB-like genes are clearly apparent in primates (Steinle et al., 1998; Seo et al., 2001) and cattle (based on est sequences in GenBank). In Sus scrofa (the pig), two MICA/B-like genes have been identified (dubbed MIC1 and MIC2) from genomic sequencing efforts (Chardon et al., 2001). At least in the pig H01 haplotype, MIC1 appears to be a pseudogene while MIC2 seems to possess features of a transcribed and functional MICA/B gene (Chardon et al., 2001).

The molecular cloning of a porcine ULBP-like cDNA and initial analysis of its functional properties are presented here. The evolution of ULBP genes and genes encoding other NKG2D ligands is an interesting aspect of the tailoring and tuning of immune responses during mammalian speciation. Characterization of genes encoding NKG2D ligands from swine may have clinical ramifications as well given the documented role of human NK cells in the rejection of porcine xenografts (Lin et al., 1997; Itescu et al., 1998). Ligands expressed by pig cells which are capable of interacting with human NKG2D potentially could be targets to minimize human NK cell-mediated killing of porcine xenografts.

\section{Materials and methods}

\subsection{DNA sequence analyses}

Sequence data was edited and assembled using the Wisconsin Package Version 10.3 (Accelrys Inc., San Diego, CA). Alignments and sequence comparisons also were performed using the Wisconsin Package. MEGA2 software (Kumar et al., 2001) was employed to create a neighbor-joining tree us- ing the proportion of synonymous and non-synonymous differences of exons 2 and 3 sequences. Bootstrap trials (10,000 repetitions) were used to determine the statistical significance of branches.

\subsection{Southern blot analyses}

Southern blot analyses were performed essentially as previously described (Crew et al., 1994). The probe was a $437 \mathrm{bp}$ fragment encompassing most of exons 2 and 3 (nucleotide positions 172-609 of GenBank AY248037) which was ${ }^{32} \mathrm{P}-$ labeled using the Random Primer kit (Invitrogen, Carlsbad, CA). Washing was at moderate stringency $\left(45^{\circ} \mathrm{C}\right.$, in $0.1 \times$ SSC, $0.1 \%$ SDS).

\subsection{RT-PCR for expression of porcine ULBP (PULBP) and porcine MIC2 (PMIC2)}

RNA from tissues of an approximately 30-day fetus (Large White-Duroc hybrid) was isolated using TRIzol (Invitrogen). RNA from AOC cells was extracted using RNeasy Mini Kit (Qiagen Inc., Valencia, CA). First strand cDNA was synthesized with SuperScript First-Strand Synthesis System for RT-PCR (Invitrogen) using random primers. Two microliters of the first strand cDNA were used to amplify the extracellular domain-encoding sequence of PULBP and PMIC2 using gene specific primers (GACGATGGCGCAGACCGCT and CGAATGGGCTATGGGGGGC for PULBP; GGTACAACTTCACGGTGATGGCCC and CACGGCGTGGACACTGTGATTCCC for PMIC2). PCR amplification of $\beta 2 \mathrm{~m}$ used ATGATATCCCACTTTTCACACCGCTCCAGTAGC and ATAGATCTGGATTCATCCAACCCAGATGCAGC as primers. PCR products were run in a $1 \%$ agarose gel.

\subsection{Porcine PBMC isolation and FACS analyses}

Porcine PBMCs were isolated by standard protocols using Ficoll-Hypaque density gradient centrifugation and monocyte depletion by adherence to plastic. Genes encoding PULBP-Fc (amino acids 1-219, seven amino acid linker, Fc of human IgG1) and HUBLP2-Fc (amino acids 1-224, two amino acid linker, $\mathrm{Fc}$ of human $\mathrm{IgG} 1$ ) fusion proteins under control of the CMV Immediate-Early promoter were constructed by standard techniques and were expressed in COS1 cells. Cell-culture supernatants were concentrated by centrifugation through Centriplus YM-10 columns (Millipore, Bedford, MA) prior to use in FACS. PE-conjugated goat antihuman IgG1 was employed as secondary antibody and was purchased from Sigma-Aldrich (St. Louis, MO).

\section{Results}

\subsection{Cloning of a porcine ULBP-like cDNA}

No entries in the limited pig EST database (only $~ 90,000$ entries) exhibited similarity with human ULBPs. Therefore, 
Table 1

Degenerate primers used for porcine ULBP cDNA cloning

F1: 5'-GACVCWCACTSTCTYTKSTAYRACWTCA-3'

F2: 5'GTGGTGTGHRGTYCARRGCCWGG-3'

F3: 5'-TTTTCTYYMCTATGACTGTG-3'

R1: 5'-CATCCTKRCCTGCAGSGTSAGRGG-3'

R2: 5'-GAACTGCCARSATCCWCKGMYGTDTCC-3'

R3: 5'-TTCTCCCACTTBTCYKTCATC-3'

we used degenerate primers corresponding to conserved regions in the human ULBP1, ULBP2, and ULBP3 (HULBP13) gene sequences in initial attempts to amplify porcine ULBP gene sequences. Three forward and three reverse degenerate primers were synthesized (Table 1) and all nine combinations were employed in PCR using pig genomic DNA from $\mathrm{PK}(15)$ cells. Only F1/R2 and F1/R3 primer pairs yielded products of the predicted sizes (data not shown). The F1/R3 PCR product (the larger fragment) was sequenced and found to contain segments with significant similarity to human ULBP1 and included intron 2 (approximately $275 \mathrm{bp}$ ). Overlapping $5^{\prime}$ and $3^{\prime}$ portions of a full length porcine ULBP cDNA from the MARC 1PIG cDNA library (Fahrenkrug et al., 2002) were obtained using primers based upon the coding sequence of this PCR product and these were subsequently ligated to yield the final full length cDNA described here. The porcine ULBP cDNA thus obtained was $1418 \mathrm{bp}$ in length and contained 93 bp $5^{\prime}$ untranslated and 574 bp $3^{\prime}$ untranslated sequences (GenBank accession number AY248037).

The predicted protein sequence encoded by the full length porcine ULBP (PULBP) cDNA is shown in Fig. 1 and is compared to HULBP1-4 protein sequences. The percent amino acid identity between PULBP and each human ULBP
Table 2

Percent amino acid identity between PULBP and human ULBP proteins

\begin{tabular}{llll}
\hline & $\alpha 1$ & $\alpha 2$ & All \\
\hline ULBP1 & 52.3 & 49.5 & 44.5 \\
ULBP2 & 48.9 & 45.2 & 39.7 \\
ULBP3 & 43.2 & 50.5 & 42.1 \\
ULBP4 & 35.2 & 43.0 & 35.2 \\
\hline
\end{tabular}

(Table 2) is comparable to the percent amino acid identities between each human ULBP (Cosman et al., 2001). PULBP is predicted to be GPI-linked (using DGPI on the ExPASy proteomics server) with a cleavage site (Fig. 1) at approximately the same relative location as HULBP1-3 (Cosman et al., 2001). The 14 residues of ULBP3 which make contact with NKG2D (Radaev et al., 2002) are relatively well conserved in PULBP-five are identical and three are conservative substitutions (Fig. 1). However, considering all the human ULBPs, 11 of the 14 residues are found in at least one of the other human ULBPs (Fig. 1).

\subsection{Phylogenetic analyses of mammalian ULBP-like genes}

The DNA sequences encoding the $\alpha 1$ and $\alpha 2$ domains of PULBP, HULBP1-4, two cattle ULBP-like gene sequences (MHCLA1 and MHCLA2, Larson et al., 2003), and the natural ligand for the murine NKG2D receptor, Raet1, were used to examine the phylogenetic relationship of mammalian ULBPlike genes. A neighbor-joining tree is shown in Fig. 2. PULBP branched more closely to the bovine ULBP-like genes, MHCLA1 and MHCLA2. Identical tree topologies were

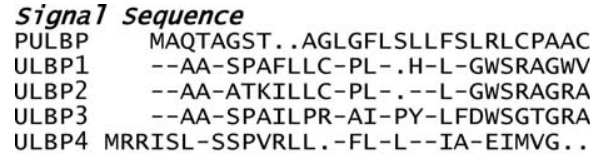

a1 Domain

PULBP DTHSLCYNFIIIPKPSPGHPWC. VQAQVDAEDFFSCDCGAAQIQSVSSLGEKVKGTKNWEIOIETLKDVGDFLKEQLPDIMPENYTAMD

ULBP1 ---C---D---T--SR-EPQ--E--GL--ERP-LHY--VNHKAKAFA---K--NV--T--E-T---R--V----G--L--QV--LIPIE

ULBP2 -P-----DITV---FR--PR--A--G---EKT-LHY---NKTVTP--P--K-LNV-TA-KA-NPV-RE-V-I-T---R--QL----PKE

ULBP3 -A---W---T--HL-RH-QQ--E--S---QKN-L-Y---SDKVL-MGH-E-QLYA-DA-GK-L-M-RE--QR-RLE-A-TEL-DF-PSG

ULBP4 .G----F--T-KSLSR--Q---EA-VFLNKNL-LQYNSDNNMVKPLGL--K--YA-ST-GELTQ--GE--RD-RML-C--K-QIK-.S-

a2 Domain

PULBP PPTLQVKVTCQSQDDGHTSGSWKFAFNGQICLHIDSDNMTLTVVHSGGRRMKEKWESDRIVTSFLRNISLGDCKSWLTHFLVHWEKMLKTAAS

ULBP1 -L---ARMS-EHEAH--GR---Q-L----KF-LF--N-RKW-AL-P-AKK-T----KN-D--M-FQK--

ULBP2 -L---ARMS-EQKAE--S----Q-S-D---F-LF--EKRMW-T--P-A-K-----N-KV-AMSFHYF-M---IG--ED--MGMDST-EPS-G

ULBP3 -L----RMS-ECEA--YIR---Q-S-D-RKF-LF--N-RKW----A-A-------K-SGL-T-FKMV-MR-----RD--M-RK-R-EPT-.

ULBP4 -S----EMF--REAERC-GA--Q--T--EKS-LF-AM---W--INHEASKI--T-KK--GLEKYF-KL-K---DH--RE--G---A-PEPTV-

\section{GPI-anchor/TM domain}

PULBP . PTTAPPIAHSKGAATKPSAWFPLLVL. VLAGLVPTVLLGV

ULBP1 KP-SL--GTTQP-AM-TTL-P-SL-IIFLCFI---R

ULBP2 APLAMSSGTTQLRAT-TTLILCCL-II-PCFI-P-I

ULBP3 .P--M--GL-QP-AI-TTL-P-SF-II-.CFI-P-I

ULBP4 ..-VN-SD-HW. .SSSSL-DR-II-GAFILL--M-I-LICVWWQNGEWQAGLWPLRTS

Fig. 1. Alignment of the predicted protein sequence of porcine ULBP (PULBP) with human ULBP protein sequences. The predicted peptide sequence of porcine ULBP (PULBP) is aligned with those of human ULBP1-4 where dashes (-) indicate identity to PULBP and alignment gaps are indicated by periods $(\cdot)$. Contact residues between human ULBP3 and human NKG2D (Radaev et al., 2002) are shaded. Conservative substitutions and non-conservative substitutions in these residues of PULBP with respect to all other human ULBPs are indicated by filled circles $(\bigcirc)$ and open circles $(\bigcirc)$, respectively. The predicted cleavage site of PULBP for GPI-linkage is noted with an arrow. Potential N-linked glycosylation sites in PULBP are underlined and in bold. 


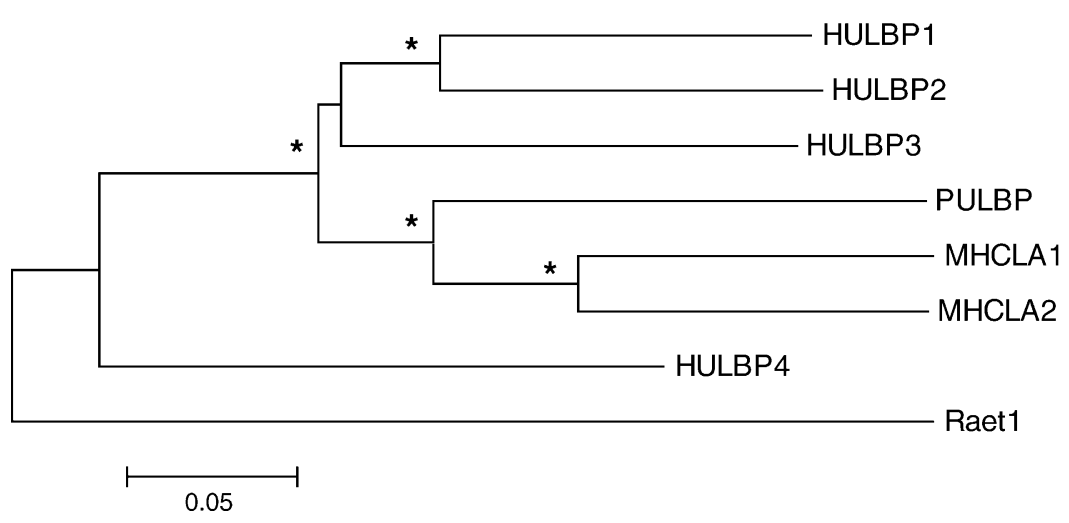

Fig. 2. Phylogenetic analysis of mammalian ULBP coding sequences. A neighbor-joining tree using the proportion ( $p$ ) of synonymous and non-synonymous differences of ULBP exons 2 and 3 sequences (encoding the $\alpha 1$ and $\alpha 2$ domains) is shown. The scale of $p$ relative to branch lengths is given by the bar below the tree. Interior branches with a bootstrap confidence level of greater than 95\% are indicated by an asterisk. Human ULBP1-3 sequences were obtained from GenBank accession numbers AF304377, AF304378, AF304379, human ULBP4 from AY252119, bovine ULBP-like DNAs (MHCLA1 and MHCLA2) from AY160681 and AF371556, and mouse Raet1 from NM_009016.

observed using a wide assortment of distance matrices. Interestingly, human ULBP4 branched outside of an intuitive human, bovine, and porcine ULBP-like gene clade suggesting that it may be of ancient origin.

\subsection{Gene copy number of porcine ULBP-like genes}

Southern blot analysis of pig genomic DNA was undertaken to roughly approximate the size of the porcine ULBP gene family. Using a $437 \mathrm{bp}$ probe (which based on human ULBP genomic sequences, was predicted to contain most of exons 2 and 3 ) and moderate stringency conditions, a single band was observed with three different restriction enzymes (Fig. 3). Identical results were obtained using the full-length cDNA as probe (data not shown). These results suggest that perhaps there is only one ULBP gene in the pig genome. If there is another ULBP gene in pigs, it is likely to be highly divergent from the PULBP sequence reported here. PCR studies using degenerate primers (Table 1) is consistent with this assertion-while several pairs of these were able to amplify at least three human ULBP gene sequences (data not shown), only one porcine ULBP-like gene was identified.

\subsection{Expression of the potential porcine $N K G 2 D$ ligands, PULBP and PMIC2}

The tissue distribution of PULBP mRNA transcripts was examined by RT-PCR. Such analyses revealed that PULBP is widely expressed with appropriately spliced transcripts detectable in all tissues examined except brain (Fig. 4A). The intestine appeared to have the highest levels of PULBP transcripts among the tissues examined. The expression of porcine MIC2 (PMIC2), another potential NKG2D ligand, was also examined in the same tissues. The sequence of PMIC2 has been previously reported (Chardon et al., 2001) but not its expression pattern. Unlike PULBP, RT-PCR analyses shows that pig MIC2 has a slightly more limited tissue distribution with transcripts detected in heart, intestine, kidney, muscle, liver, lung, testes, and thymus (Fig. 4A). PMIC2 transcripts were not detected in PBMC or spleen.

Basal and inducible expression of PULBP and PMIC2 was also examined in AOC cells, a porcine aortic endothelial cell line (Carrillo et al., 2002). Although not a quantitative assay,

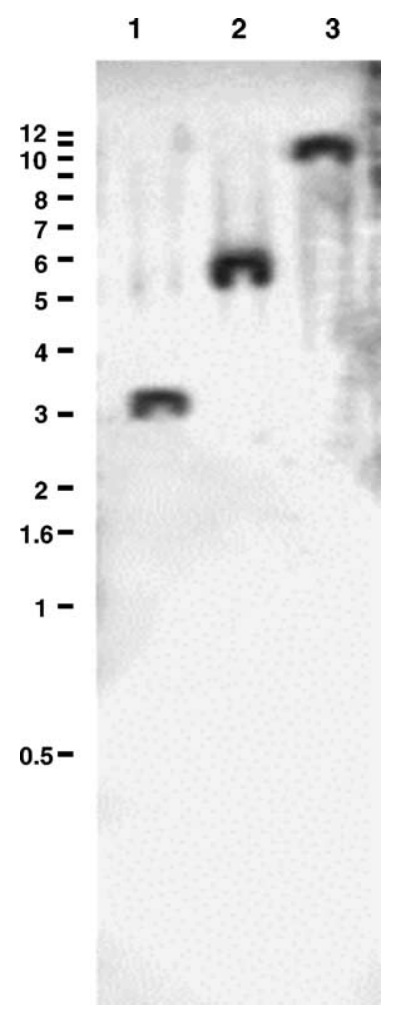

Fig. 3. Southern blot analysis of porcine ULBP gene copy number. Pig genomic DNA $(20 \mu \mathrm{g}$, from LLC-PK1 cells) cleaved with BamHI, EcoRI, or HindIII (lanes 1-3, respectively), was analyzed by Southern blotting using a 437 bp fragment of PULBP cDNA as a probe as described in Section 2. Positions corresponding to migration of DNA size standards (in kbs) are noted at the left of the autoradiogram. 


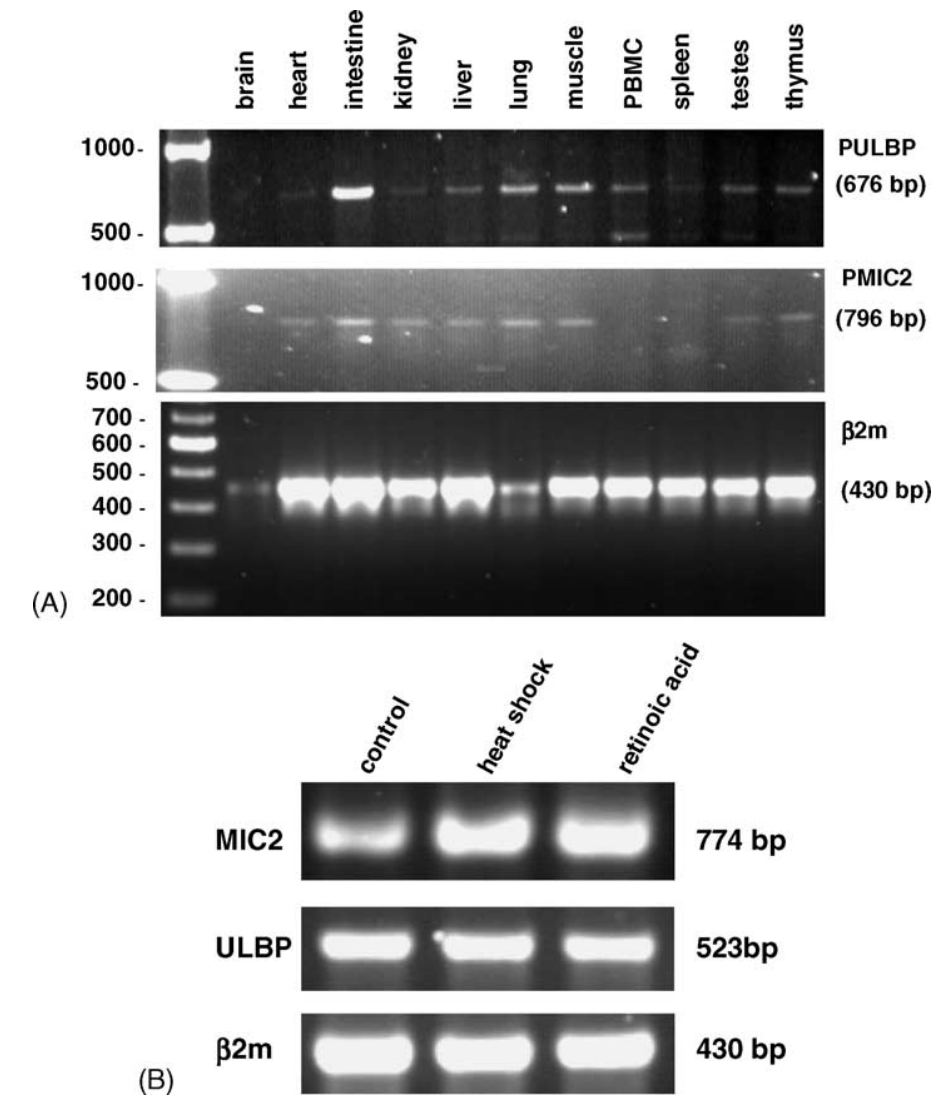

Fig. 4. Expression of potential porcine ligands for NKG2D. (A) Tissue distribution of PULBP and PMIC2 transcripts. Gene-specific primers for PULBP, PMIC2, and $\beta 2 \mathrm{~m}$ were used in PCR using first strand cDNA reverse transcribed from RNA of the indicated pig tissues. The size of correctly spliced transcripts is indicated at the right. (B) Inducible expression of PULBP in a porcine aortic endothelial cell line. RT-PCR using primers specific for pig ULBP, MIC2, and, as a control, $\beta 2 \mathrm{~m}$ (as indicated at the left) was carried out using RNA from control cells or cells induced with heat shock ( $42^{\circ} \mathrm{C}$ for $\left.90 \mathrm{~min}\right)$ or $1 \mathrm{uM}$ all-trans retinoic acid (as indicated above the lanes). Primers for PULBP amplification were CGATACTCACTCTCTTTGC and CGCTGTCTTCAACATTTTCTCCC. The predicted size of correctly spliced cDNA (indicated at the right) was observed in all nine RT-PCR reactions.

PMIC2 transcript levels appeared to be increased by both heat shock and retinoic acid (Fig. 4B) PULBP expression, though readily detectable in AOC cells, appeared unaffected by either treatment (Fig. 4B).

\subsection{Binding of PULBP-FC recombinant protein to pig PBMCs}

As a first step in the functional characterization of PULBP, we sought to determine whether the PULBP protein is able to bind a subset of lymphocytes as does its human orthologs and whether PULBP is able to bind human NKG2D. To this end a gene encoding a PULBP-Fc (IgG1) fusion protein was constructed and expressed in COS- 1 cells. The media from such cultures was concentrated and used for FACS analyses of porcine PBMCs and a human NK cell line, NKL cells (chosen because they uniformly express NKG2D). A human ULBP2-Fc fusion protein was examined as well. As a negative control, cell culture supernatants from COS-1 cells expressing a GFP-Fc fusion protein were employed. The results, shown in Fig. 5, suggests that PULBP is able to bind a portion of porcine PBMCs although the nature of this subset could pig PBMCs
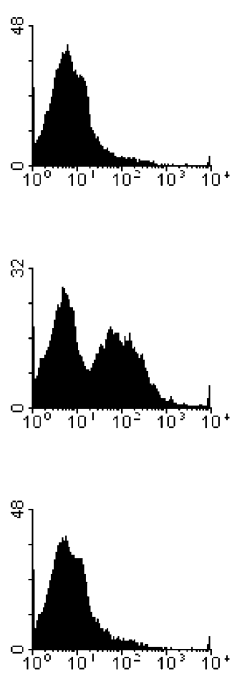

NKL cells
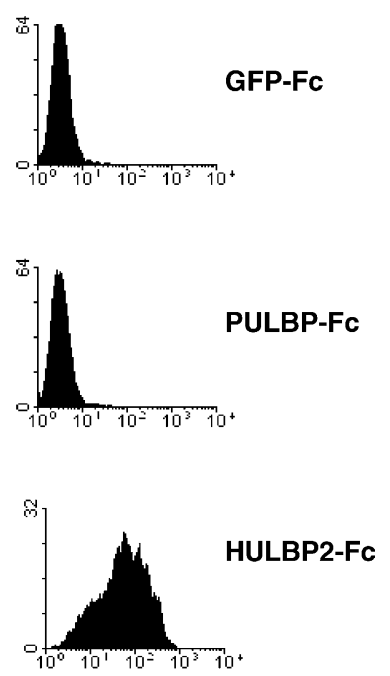

Fig. 5. Binding of PULBP-Fc fusion protein to porcine PBMCs. FACS analyses of porcine PBMCs and a human NK cell line (NKL cells) using a GFP-Fc fusion protein (as negative control), PULBP-Fc, and HULBP2-Fc (indicated at the right of the histograms) are shown. 
not be determined because of the lack of appropriate antibodies to porcine cell-surface markers. Interestingly, human ULBP2 exhibited no binding to porcine PBMCs. Conversely, no binding to NKL cells was observed with PULBP-Fc although binding of HULBP2-Fc to NKL cells was quite evident (Fig. 5).

\section{Discussion}

We have cloned a porcine homolog of human ULBPs denoted here as PULBP. PULBP exhibits $35-52 \%$ amino acid identity to human ULBPs (Table 1) and phylogenetic analyses places PULBP in close evolutionary relationship to the bovine ULBP-like genes MHCLA1 and MHCLA2 (Fig. 2). Scrutiny of the predicted PULBP amino acid sequence with respect to residues making contact with NKG2D (Radaev et al., 2001) revealed a relatively high level of amino acid conservation at these positions (Fig. 1).

Southern blot results (Fig. 3) suggest that only one PULBP exists in the pig genome. Even at low stringency hybridization using full-length PULBP as probe, only one band was observed. We conclude that if other ULBP-like genes exist in the pig genome, they are significantly divergent from the PULBP cDNA described here. If, in fact, there is only one porcine ULBP gene and the pseudogene features of porcine MIC1 in the H01 haplotype (Chardon et al., 2001) are found in all pig haplotypes, then the pig apparently expresses only two NKG2D ligands. This is in sharp contrast to the at least six expressed by humans.

Similar to human ULBPs, transcripts of PULBP were observed in a variety of tissues (Fig. 4A). The relatively high level of PMIC2 expression observed in the intestine (Fig. 4A) is consistent with previous studies of human MICA/B as well (Groh et al., 1996). Furthermore, human MICA/B are inducible by heat shock and retinoic acid (Groh et al., 1996; Jinushi et al., 2003) and PMIC2, but not PULBP, transcripts were increased by these treatments as well (Fig. 4B). Whether or not the presence of PULBP or PMIC2 transcripts is indicative of cell-surface expression must await the development of antibodies specific for these proteins.

A striking aspect of ligands for human NKG2D is their diversity both in terms of sequence variation and domain composition. An induced fit model, whereby NKG2D adopts local conformational changes upon ligand interaction, has been proposed as the structural basis for the ability of NKG2D to bind such a diverse array of ligands (Radaev et al., 2001). Using the crystal structure coordinates of NKG2D complexed with different ligands, Radaev et al. (2002) developed an algorithm to score potential ligands of NKG2D (http://red.niaid.gov/programs). The receptor:ligand algorithm evaluates abilities to form interface salt bridges, hydrogen bonds, and hydrophobic interactions, calculates the solvent accessibility weighted energetic contribution of each interface residue, and then figures ligand compatibility for the NKG2D receptor based on a mutational matrix. Two different templates of the interface can be assumed in the algorithm, one using the human ULBP3/NKG2D coordinate data and the other using the coordinates of the mouse Rae1b/NKG2D structure. Using this algorithm with the ULBP3 template, PULBP scored higher than human ULBP1 and ULBP2 (data not shown) suggesting that PULBP is a compatible ligand for human NKG2D. It was, therefore, surprising to observe that PULBP, although capable of binding porcine PBMCs, was unable to bind to human NK cells and that human ULBP2 which was able to bind human NK cells could not stably interact with porcine PBMCs (Fig. 5). Thus, while human NKG2D can be considered promiscuous in ligand binding, this promiscuity apparently does not extend across species. PULBP has three potential $\mathrm{N}$-linked glycosylation sites (Fig. 1), one of which corresponds to an amino acid that is important in the interaction between human NKG2D and ULBP3 (Radaev et al., 2002). It is possible that this glycosylation could prevent the binding of PULBP to human NKG2D.

Pig NKG2D has $67 \%$ amino acid identity with human NKG2D (Yim et al., 2002) and we propose that the crossspecies difference in NKG2D primary structure accounts for the cross-species incompatibility of ULBP-NKG2D interaction. Whether this incompatibility extends to other NKG2D ligands, other human ULBPs or MIC-like proteins, is currently under investigation. An mAb against human NKG2D substantially blocked human NK cell-mediated lysis of a porcine aortic endothelial cell line (unpublished data) suggesting that there is at least one pig NKG2D ligand capable of binding to human NKG2D.

\section{Acknowledgements}

This work was supported in part by the Office of Research and Development, Department of Veterans Affairs and NIH AI49885 (M.D.C.), AI054324 (C.N.G.-B.) and by the National Center for Research Resources grants through the BRIN Program (P20 RR-16460). Thanks go to Dr. Tim Smith (Meat Animal Research Center, Omaha, Nebraska) for providing the MARC 1PIG cDNA library.

\section{References}

Carrillo, A., Chamorro, S., Rodriguez-Gago, M., Alvarez, B., Molina, M.J., Rodriguez-Barbosa, J.I., Sanchez, A., Ramirez, P., Munoz, A., Dominguez, J., Parrilla, P., Yelamos, J., 2002. Isolation and characterization of immortalized porcine aortic endothelial cell lines. Vet. Immunol. Immunopathol. 89, 91-98.

Cerwenka, A., Bakker, A.B., McClanahan, T., Wagner, J., Wu, J., Phillips, J.H., Lanier, L.L., 2000. Retinoic acid early inducible genes define a ligand family for the activating NKG2D receptor in mice. Immunity 12, 721-727.

Cerwenka, A., O'Callaghan, C.A., Hamerman, J.A., Yadav, R., Ajayi, W., Roopenian, D.C., Joyce, S., Lanier, L.L., 2002. Cutting edge: the minor histocompatibility antigen $\mathrm{H} 60$ peptide interacts with both $\mathrm{H}-2 \mathrm{~Kb}$ and NKG2D. J. Immunol. 168, 3131-3134. 
Chardon, P., Rogel-Gaillard, C., Cattolico, L., Duprat, S., Vaiman, M., Renard, C., 2001. Sequence of the swine major histocompatibility complex region containing all non-classical class I genes. Tissue Antigens 57, 55-65.

Cosman, D., Müllberg, J., Sutherland, C., Chin, W., Armitage, R., Fanslow, W., Kubin, M., Jan, N., 2001. ULBPs, novel MHC classs Irelated molecules, bind to CMV glycoprotein UL16 and stimulate NK cytotoxicity through the NKG2D receptor. Immunity 14, 123-133.

Crew, M.D., Douglass, C.A., Brorson, K.A., 1994. H-2T24 and PemaT24: orthologous expressed MHC class Ib genes from mouse and Peromyscus maniculatus. Immunogenetics 39, 412-417.

Diefenbach, A., Jensen, E.R., Jamieson, A.M., Raulet, D.H., 2001. Rae1 and $\mathrm{H} 60$ ligands of the NKG2D receptor stimulate tumour immunity. Nature 413, 165-171.

Fahrenkrug, S.C., Smith, T.P., Freking, B.A., Cho, J., White, J., Vallet, J., Wise, T., Rohrer, G., Pertea, G., Sultana, R., Quackenbush, J., Keele, J.W., 2002. Porcine gene discovery by normalized cDNA-library sequencing and EST cluster assembly. Mamm. Genome 13, 475478.

Groh, V., Bahram, S., Bauer, S., Herman, A., Beauchamp, M., Spies, T., 1996. Cell stress-regulated human major histocompatibility complex class I gene expressed in gastrointestinal epithelium. Proc. Natl. Acad. Sci. U.S.A. 93, 12445-12450.

Groh, V., Rhinehart, R., Secrist, H., Bauer, S.K., Grabstein, H., Spies, T., 1999. Broad tumor-associated expression and recognition by tumorderived $\gamma \delta \mathrm{T}$ cells of MICA and MICB. Proc. Natl. Acad. Sci. U.S.A. 96, 6879-6884.

Groh, V., Rhinehart, R., Randolph-Habecker, J., Topp, M.S., Riddell, S.R., Spies, T., 2001. Costimulation of CD8 $\alpha \beta$ T cells by NKG2D via engagement by MIC induced on virus-infected cells. Nat. Immunol. 2, 255-260.

Itescu, S., Kwiatkowski, P., Artrip, J.H., Wang, S.F., Ankersmit, J., Minanov, O.P., Michler, R.E., 1998. Role of natural killer cells, macrophages, and accessory molecule interactions in the rejection of pig-to-primate xenografts beyond the hyperacute period. Hum. Immunol. 59, 275-286.

Jan, C.N., Sutherland, C.L., Lawrence, W.A., Rein-Weston, A., Cosman, D., 2003. ULBP4 is a novel ligand for human NKG2D. Biochem. Biophys. Res. Commun. 305, 129-135.

Jinushi, M., Takehara, T., Tatsumi, T., Kanto, T., Groh, V., Spies, T. Kimura, R., Miyagi, T., Mochizuki, K., Sasaki, Y., Hayashi, N., 2003.
Expression and role of MICA and MICB in human hepatocellular carcinomas and their regulation by retinoic acid. Int. J. Cancer 104, 354-361.

Kumar, S., Tamura, K., Jakobsen, I.B., Nei, M., 2001. MEGA2: molecular evolutionary genetics analysis software. Bioinformatics 17 , 1244-1245.

Larson, J.H., Rebeiz, M.J., Stiening, C.M., Windish, R.L., Beever, J.E., Lewin, H.A., 2003. MHC class I-like genes in cattle, MHCLA, with similarity to genes encoding $\mathrm{NK}$ cell stimulatory ligands. Immunogenetics 55, 16-22.

Lin, Y., Vandeputtte, M., Waer, M., 1997. Natural killer cell- and macrophage-mediated rejection of concordant xenografts in the absence of $\mathrm{T}$ and B cell responses. J. Immunol. 158, 5658-5667.

Radaev, S., Rostro, B., Brooks, A.G., Colonna, M., Sun, P.D., 2001. Conformational plasticity revealed by the cocrystal structure of NKG2D and its class I MHC-like ligand ULBP3. Immunity 15, 1039-1049.

Radaev, S., Kattah, M., Zou, Z., Colonna, M., Sun, P., 2002. Making sense of the diverse ligand recognition by NKG2D. J. Immunol. 169, 6279-6285.

Rolle, A., Mousavi-Jazi, M., Eriksson, M., Odeberg, J., SoderbergNaucler, C., Cosman, D., Karre, K., Cerboni, C., 2003. Effects of human cytomegalovirus infection on ligands for the activating NKG2D receptor of NK cells: up-regulation of UL16-binding protein (ULBP)1 and ULBP2 is counteracted by the viral UL16 protein. J. Immunol. 171, 902-908.

Seo, J.W., Walter, L., Gunther, E., 2001. Genomic analysis of MIC genes in rhesus macaques. Tissue Antigens 2001, 159-165.

Steinle, A., Groh, V., Spies, T., 1998. Diversification, expression, and gamma delta $\mathrm{T}$ cell recognition of evolutionarily distant members of the MIC family of major histocompatibility complex class I-related molecules. Proc. Natl. Acad. Sci. U.S.A. 95, 12510-12515.

Sutherland, C., Jan, N., Schooley, K., VandenBos, T., Kubin, M., Cosman, D., 2002. UL16-binding proteins, novel MHC class I-related proteins, bind to NKG2D and activate multiple signaling pathways in primary NK cells. J. Immunol. 168, 671-679.

Vivier, E., Tomasello, E., Paul, P., 2002. Lymphocyte activation via NKG2D: towards a new paradigm in immune recognition? Curr. Opin. Immunol. 14, 306-311.

Yim, D., Sotiriadis, J., Kim, K.S., Shin, S.C., Jie, H.B., Rothschild, M.F., Kim, Y.B., 2002. Molecular cloning, expression pattern and chromosomal mapping of pig CD69. Immunogenetics 54, 276-281. 